\title{
Dynamics of cancer cell subpopulations in primary and metastatic colorectal tumors
}

\author{
Teodora Evgenieva Goranova • Masayuki Ohue • \\ Yutaro Shimoharu $\cdot$ Kikuya Kato
}

Received: 23 December 2010/Accepted: 17 February 2011/Published online: 5 March 2011

(C) The Author(s) 2011. This article is published with open access at Springerlink.com

\begin{abstract}
Intratumor heterogeneity-heterogeneity of cancer cells within a single tumor-is considered one of the most problematic factors of treatment. Genetic heterogeneity, such as in somatic mutations and chromosome aberrations, is a common characteristic of human solid tumors and is probably the basis of biological heterogeneity. Using mutations in $A P C, T P 53$ and KRAS as markers to identify distinct colorectal cancer subpopulations, we analyzed a total of 42 primary colorectal cancer tissues and six paired liver metastases with multipoint microsampling, which enabled analysis of mutation patterns and allelic imbalances with a resolution of $0.01 \mathrm{~mm}^{2}$ (about 200 cells). There was usually more than one subpopulation in each primary tumor. Only two of $15(13.3 \%)$ cases with three gene mutations and eight of 27 (29.6\%) cases with two gene mutations had a single subpopulation. Cells
\end{abstract}

Electronic supplementary material The online version of this article (doi:10.1007/s10585-011-9381-0) contains supplementary material, which is available to authorized users.

T. E. Goranova · Y. Shimoharu · K. Kato $(\bowtie)$

Research Institute, Osaka Medical Center for Cancer

and Cardiovascular Diseases, 1-3-3 Nakamichi,

Higashinari-ku, Osaka 537-8511, Japan

e-mail: katou-ki@mc.pref.osaka.jp

M. Ohue

Department of Surgery, Osaka Medical Center for Cancer and Cardiovascular Diseases, 1-3-3 Nakamichi,

Higashinari-ku, Osaka 537-8511, Japan

Present Address:

T. E. Goranova

Molecular Medicine Center, 2 Zdrave Street,

1431 Sofia, Bulgaria with mutations in all of the examined genes usually constituted the major population. Multipoint microsampling of six primary and metastatic tumor pairs revealed that the majority of discrepancies in mutation patterns found with the bulk tissue analysis were due to loss of subpopulations in the metastatic tissues. In addition, multipoint microsampling uncovered substantial changes in subpopulations that were not detected with bulk tissue analysis. Specifically, the proportion of KRAS mutation-negative subpopulations increased in the metastatic tumors of four cases. Because KRAS mutation status is linked to cetuximab/panitumumab efficacy, subpopulation dynamics could lead to differences in response to cetuximab/panitumumab in primary versus metastatic tumors.

Keywords Intratumor heterogeneity - Molecular target drug $\cdot$ Colorectal cancer $\cdot$ Somatic mutation

\author{
Abbreviations \\ CSC Cancer stem cell \\ SAP Shrimp alkaline phosphatase
}

\section{Introduction}

Intratumor heterogeneity, which is the heterogeneity of cancer cells within a single tumor, is considered one of the most problematic factors of treatment. During anti-cancer therapy, the initial regression of the tumor eventually leads to the outgrowth of drug-resistant cells. Resistant cells likely exist in a heterogeneous primary cancer cell population rather than evolve from it. A recently emerging topic related to heterogeneity is the cancer stem cell (CSC) hypothesis [1], which assumes that only a fraction of cancer cells have the 
ability to initiate tumor formation. In addition, the CSC hypothesis claims that currently available drugs are not effective because they target the total cancer cell population.

In addition to the heterogeneity of biological features presented by CSC, genetic heterogeneity, such as in somatic mutations and chromosome aberrations, is a common characteristic of human solid tumors and is probably the basis of biological heterogeneity. A number of studies have described genetic heterogeneity [2-11]. Although these studies have demonstrated that cancer cell subpopulations with different mutation patterns are present in most solid tumors, there were several technical limitations. In particular, these studies did not use laser microdissection, which is now a standard technique, and they had a relatively high threshold of detection, which may have hampered the detection of rare clones.

Genetic heterogeneity within primary tumors is a single aspect of tumor heterogeneity. Although tumors develop at one site, some cancer cells leave the primary tumor and develop metastases at distant sites. Because metastases account for the majority of cancer-related deaths, understanding the underlying mechanism of their development is extremely important. Analysis of genetic heterogeneity, such as in somatic mutations, may help clarify the issue. Although intratumor heterogeneity has been intensely studied, the question of heterogeneity within metastases remains unexplored [12].

Colorectal cancer is one of the most common and wellstudied cancer types. Because somatic mutations in the $A P C, K R A S$ and TP53 genes have been established as "drivers" for colorectal carcinogenesis [13], we chose these genes as markers to identify cancer cell subpopulations. We analyzed a total of 42 primary colorectal cancer tissues and 6 paired metastatic tissues with multipoint microsampling $[11,14]$, which is our proprietary technical approach for analyzing intratumor genetic heterogeneity. Multipoint microsampling enables analysis of mutation patterns and allelic imbalance with a resolution of $0.01 \mathrm{~mm}^{2}$ (about 200 cells). We examined the prevalence of intratumor genetic heterogeneity in primary tumors and the dynamics of cancer cell subpopulations in primary and metastatic tumors.

\section{Materials and methods}

\section{Samples}

In a previous study, a total of 86 bulk primary colorectal cancer tissues from our tumor tissue bank were examined for mutations in the APC, KRAS and TP53 genes. DNA was extracted from frozen bulk tumor tissues using the QIAamp DNA Micro Kit (Qiagen, Hilden, Germany), and the coding regions of $A P C, K R A S$ and TP53 were examined for mutations using High-Resolution Melting on a LightScanner (Idaho Technology, Salt Lake City, UT, USA). Samples with aberrant melting curves were analyzed using direct sequencing with the BigDye Terminator Cycle Sequencing Kit (ver. 3.1, Applied Biosystems, Carlsbad, CA, USA) on an ABI PRISM 3730 (Applied Biosystems). Cases with a single mutation, in which cells with no mutation were the only heterogeneous subpopulation, were excluded because such a subpopulation was difficult to distinguish from normal epithelial cells. Forty-two primary colorectal cancer tissues were included in the present study: 15 tumor tissues with mutations in all three genes (i.e., APC, KRAS and TP53 genes) and 27 tumors with only two mutated genes. The details of the mutations in these tumors are listed in Table S1. In addition, 6 liver metastatic tissues from the cases with mutations in all three genes were included in the study. The present study was approved by the Ethical Committee of the Osaka Medical Center for Cancer and Cardiovascular Diseases, and informed consent was obtained from all patients.

\section{Multipoint microsampling}

For the analysis of intratumor heterogeneity, $40-\mu \mathrm{m}$-thick sections from frozen cancer tissues were prepared on a Leica CM1900 cryostat (Leica Microsystems, Wetzlar, Germany) and stained with Mayer's hematoxylin (Wako, Osaka, Japan). Forty to fifty small areas $(100 \times 100 \mu \mathrm{m})$ containing only tumor cells were microdissected from each sample. Microdissection was performed using the Leica AS LMD system (Leica Microsystems). The sampling was randomized, but we avoided repeated sampling from the same region. Genomic DNA was extracted using the prepGEM Kit (ZyGEM, Hamilton, New Zealand), according to the manufacturer's protocol, and a 20- $\mu$ l DNA mixture was prepared from each sample.

\section{PCR amplification and SNaPshot assay}

For each case, the DNA fragments that were found to contain mutations in bulk tissue analysis were simultaneously amplified using multiplex PCR on a GeneAmp PCR System 9700 (Applied Biosystems). The PCR was performed in a $10-\mu \mathrm{l}$ reaction volume including $5 \mu \mathrm{l}$ DNA (approximately $250 \mathrm{pg}$ ), 1× PCR buffer (Applied Biosystems), $2 \mathrm{mM}$ $\mathrm{MgCl}_{2}, 200 \mu \mathrm{M}$ of each dNTP, $0.2 \mu \mathrm{M}$ of each primer (Table S2) and $1 \mathrm{U}$ AmpliTaqGold polymerase (Applied Biosystems). The cycling conditions consisted of an initial denaturation step at $94^{\circ} \mathrm{C}$ for $5 \mathrm{~min}, 40$ cycles of denaturing at $94^{\circ} \mathrm{C}$ for $30 \mathrm{~s}$, annealing at $54-56^{\circ} \mathrm{C}$ for $30 \mathrm{~s}$, extension at $72^{\circ} \mathrm{C}$ for $40 \mathrm{~s}$ and a final synthesis at $72^{\circ} \mathrm{C}$ for $5 \mathrm{~min}$.

The mutation status was quantitatively determined using the SNaPshot assay. Each primer was designed to bind to a complementary template immediately adjacent to the 
mutation site. The reaction was carried out in the presence of fluorescently labeled ddNTPs, and DNA polymerase was used to extend the primer by one nucleotide (adding a single ddNTP to its $3^{\prime}$ end). The following fluorescent dyes were used for the dideoxynucleotides: A, dR6G; C, dTAMRA; G, dR110; and T, dROX.

The PCR fragments were prepared for primer extension by incubating a mixture containing $7.5 \mu \mathrm{l}$ PCR product, $0.5 \mathrm{U}$ shrimp alkaline phosphatase (SAP) (TaKaRa, Otsu, Japan) and $1 \mathrm{U}$ exonuclease I (TaKaRa) in a final volume of $10 \mu \mathrm{l}$ at $37^{\circ} \mathrm{C}$ for $40 \mathrm{~min}$, which was followed by inactivation of the enzymes at $80^{\circ} \mathrm{C}$ for $20 \mathrm{~min}$. Primer extension was carried out in a $5-\mu 1$ reaction, which contained $2 \mu \mathrm{l}$ treated PCR product, $2.5 \mu \mathrm{l}$ ABI Prism SNaPshot Multiplex Ready Reaction Mix (Applied Biosystems) and $0.5 \mu \mathrm{l}$ extension primer mix $(0.2 \mu \mathrm{M}$ of each primer). The primer sequences are shown in Table S2. The cycling conditions, which were carried out according to the manufacturer's protocol, included 25 cycles of denaturation at $96^{\circ} \mathrm{C}$ for $10 \mathrm{~s}$, annealing at $50^{\circ} \mathrm{C}$ for $5 \mathrm{~s}$ and extension at $60^{\circ} \mathrm{C}$ for $30 \mathrm{~s}$. To remove the unincorporated ddNTPs, $5 \mu \mathrm{l}$ of the SNaPshot products was incubated for $40 \mathrm{~min}$ at $37^{\circ} \mathrm{C}$ with $0.5 \mathrm{U}$ SAP (TaKaRa) in a final volume of $6 \mu \mathrm{l}$, and the enzyme was inactivated as described above. A $1-\mu 1$ aliquot of the treated SNaPshot reaction was denatured in $9 \mu \mathrm{l}$ distilled water in the presence of GeneScan 120 LIZ Size Standard (Applied Biosystems) for $5 \mathrm{~min}$ at $95^{\circ} \mathrm{C}$ and analyzed on an ABI PRISM 3100 Genetic Analyzer
(Applied Biosystems). Fragment analysis was performed with Gene Mapper Software v4.0 (Applied Biosystems).

Data analysis

The mutation status of each area was determined by calculating the mutant allele ratio $(\Delta \mathrm{M})$ for each gene using the following equation: $\Delta M=M /(M+N)$, where $M$ is the mutant allele peak height and $\mathrm{N}$ is the normal peak height. The reproducibility of amplification and the SNaPshot assay was confirmed in our previous experiments [14]. In most cases of mutant allele loss, the corresponding peak height (M) was zero. However, there were several areas with residual peaks where we set the threshold for $\Delta \mathrm{M}$ at 0.05 .

\section{Results}

Intratumor heterogeneity of primary colorectal cancers

In a previous study, we established a method called multipoint microsampling to characterize genetic heterogeneity in colorectal cancer using laser microdissection [11, 14]. This method has two characteristics: (1) a sample size small enough to keep contamination from other cell populations to a minimum, but large enough to ensure unbiased amplification, and (2) a high enough sample number $(\sim 50)$ to obtain reproducible results from minor
Fig. 1 An example of intratumor heterogeneity. a A microscopic view of a colorectal cancer tissue section with black circles indicating the microdissected areas (1-5). b Electropherograms of the SNaPshot assay. The first two peaks represent the mutation status of $A P C(\mathrm{C}>\mathrm{G})$, and the second two peaks represent the mutation status of $K R A S(\mathrm{G}>\mathrm{A})$. The blue peak is a fragment amplified with ddG, the black peak is a fragment amplified with $\mathrm{ddC}$ and the green peak is a fragment amplified with ddA. c Graphic representation of the SNaPshot results. The mutant allele ratio $(\triangle \mathrm{M})$ of $A P C$ is plotted on the $x$-axis, and the $\triangle \mathrm{M}$ of $K R A S$ is plotted on the $y$-axis
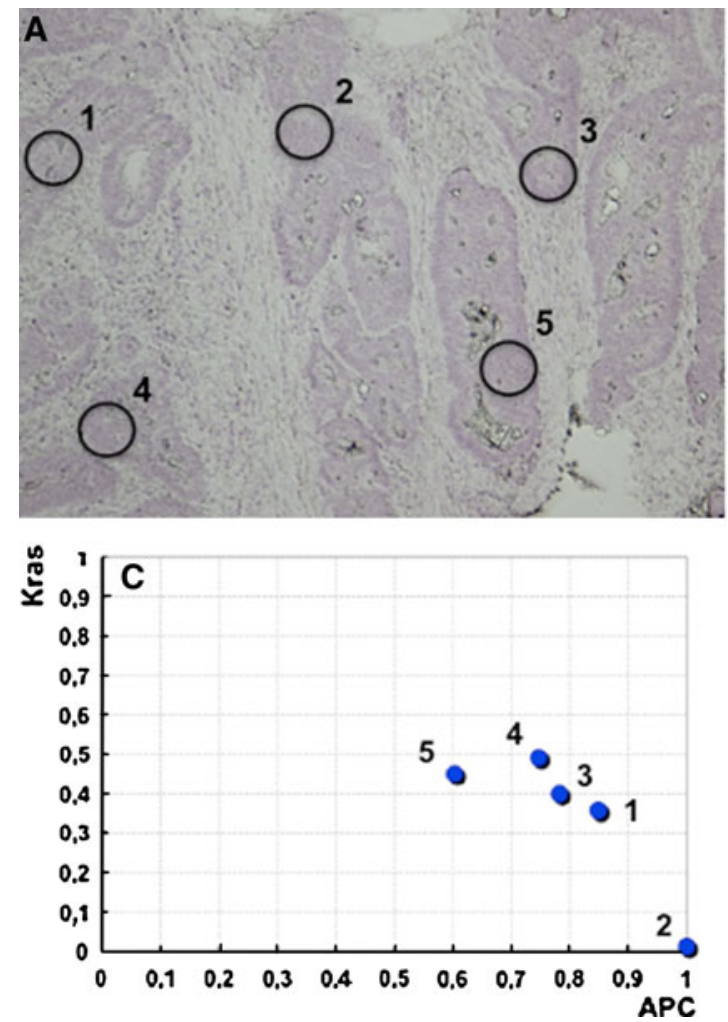
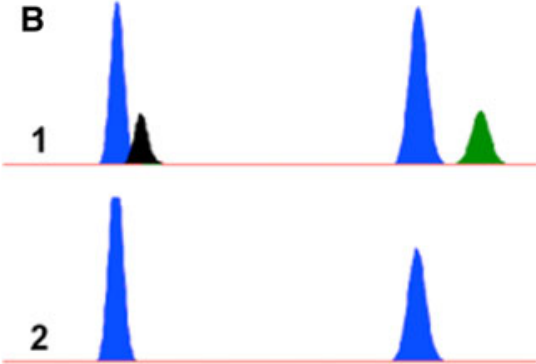

4

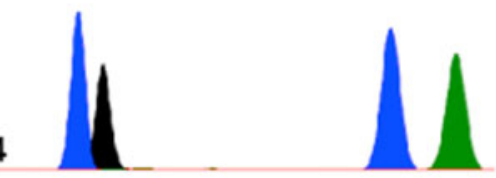

5 
Table 1 Summary of the genotypes in the 42 primary tumors

\begin{tabular}{|c|c|c|c|c|c|c|c|c|c|c|c|}
\hline \multirow[t]{2}{*}{ Case } & \multirow[t]{2}{*}{ Clinical stage } & \multirow[t]{2}{*}{ Samples/tumor } & \multirow[t]{2}{*}{ Clone } & \multicolumn{3}{|l|}{ Genotype } & \multirow[t]{2}{*}{ Areas } & \multirow[t]{2}{*}{$\%$} & \multirow[t]{2}{*}{$\mathrm{AIH}^{\mathrm{b}}-\mathrm{APC}$} & \multirow[t]{2}{*}{$\mathrm{AIH}^{\mathrm{b}}-\mathrm{KRAS}$} & \multirow[t]{2}{*}{$\mathrm{AIH}^{\mathrm{b}}-\mathrm{TP} 53$} \\
\hline & & & & $A P C$ mut $^{\mathrm{a}}$ & $K R A S$ mut $^{\mathrm{a}}$ & $T P 53 \mathrm{mut}^{\mathrm{a}}$ & & & & & \\
\hline \multirow[t]{3}{*}{1} & \multirow[t]{3}{*}{ IV } & \multirow[t]{3}{*}{42} & $\mathrm{~A}$ & - & - & + & 3 & 7,1 & + & + & + \\
\hline & & & B & + & - & + & 1 & 2,4 & & & \\
\hline & & & $\mathrm{C}$ & + & + & + & 38 & 90,5 & & & \\
\hline \multirow[t]{2}{*}{3} & \multirow[t]{2}{*}{ II } & \multirow[t]{2}{*}{48} & A & - & + & + & 2 & 4,2 & + & + & + \\
\hline & & & $\mathrm{B}$ & + & + & + & 46 & 95,8 & & & \\
\hline \multirow[t]{3}{*}{11} & \multirow[t]{3}{*}{ III } & \multirow[t]{3}{*}{41} & $\mathrm{~A}$ & + & - & - & 7 & 17,1 & + & + & - \\
\hline & & & $\mathrm{B}$ & + & + & - & 4 & 9,8 & & & \\
\hline & & & $\mathrm{C}$ & + & + & + & 30 & 73,2 & & & \\
\hline \multirow[t]{2}{*}{19} & III & 45 & $\mathrm{~A}$ & - & + & + & 2 & 4,4 & + & + & + \\
\hline & & & $\mathrm{B}$ & + & + & + & 43 & 95,6 & & & \\
\hline 24 & II & 49 & A & + & - & + & 2 & 4,1 & + & + & + \\
\hline & & & $\mathrm{B}$ & + & + & + & 47 & 95,9 & & & \\
\hline 33 & IV & 48 & A & + & - & - & 21 & 43,8 & - & + & + \\
\hline & & & B & + & + & - & 9 & 18,8 & & & \\
\hline & & & $\mathrm{C}$ & + & + & + & 18 & 37,5 & & & \\
\hline 41 & III & 40 & A & - & - & + & 7 & 17,5 & + & + & + \\
\hline & & & B & - & + & + & 1 & 2,5 & & & \\
\hline & & & $\mathrm{C}$ & + & + & + & 32 & 80 & & & \\
\hline $49^{c}$ & IV & 40 & A & $+/+$ & + & + & 40 & 100 & + & + & - \\
\hline 51 & IV & 42 & A & - & - & + & 1 & 2,4 & + & + & + \\
\hline & & & B & + & - & + & 2 & 4,8 & & & \\
\hline & & & $\mathrm{C}$ & + & + & + & 39 & 92,9 & & & \\
\hline 65 & IV & 49 & A & + & - & - & 2 & 4,1 & + & + & + \\
\hline & & & B & + & - & + & 2 & 4,1 & & & \\
\hline & & & $\mathrm{C}$ & + & + & + & 45 & 91,8 & & & \\
\hline 74 & I & 41 & A & + & + & + & 41 & 100 & + & - & + \\
\hline 81 & IV & 42 & A & + & + & - & 4 & 9,5 & + & + & + \\
\hline & & & B & + & + & + & 38 & 90,5 & & & \\
\hline 82 & IV & 48 & A & + & - & + & 1 & 2,1 & + & + & + \\
\hline & & & B & - & - & + & 3 & 6,3 & & & \\
\hline & & & $\mathrm{C}$ & - & + & + & 41 & 85,4 & & & \\
\hline & & & $\mathrm{D}$ & + & + & + & 1 & 2,1 & & & \\
\hline 83 & IV & 45 & A & + & + & - & 4 & 8,9 & + & + & + \\
\hline & & & B & + & + & + & 41 & 91,1 & & & \\
\hline 85 & IV & 44 & A & + & - & + & 1 & 2,3 & + & + & + \\
\hline & & & B & - & + & - & 2 & 4,5 & & & \\
\hline & & & $\mathrm{C}$ & - & - & + & 1 & 2,3 & & & \\
\hline & & & $\mathrm{D}$ & - & + & + & 35 & 79,5 & & & \\
\hline & & & $\mathrm{E}$ & + & + & + & 5 & 11,4 & & & \\
\hline 4 & III & 44 & A & + & + & - & 44 & 100 & + & + & ND \\
\hline 6 & II & 40 & A & + & + & - & 40 & 100 & - & - & ND \\
\hline 12 & III & 49 & A & + & - & - & 13 & 26,5 & + & + & ND \\
\hline & & & B & + & + & - & 36 & 73,5 & & & \\
\hline 13 & II & 45 & A & - & + & - & 1 & 2,2 & + & + & ND \\
\hline & & & B & + & + & - & 44 & 97,8 & & & \\
\hline
\end{tabular}


Table 1 continued

\begin{tabular}{|c|c|c|c|c|c|c|c|c|c|c|c|}
\hline \multirow[t]{2}{*}{ Case } & \multirow[t]{2}{*}{ Clinical stage } & \multirow[t]{2}{*}{ Samples/tumor } & \multirow[t]{2}{*}{ Clone } & \multicolumn{3}{|l|}{ Genotype } & \multirow[t]{2}{*}{ Areas } & \multirow[t]{2}{*}{$\%$} & \multirow[t]{2}{*}{$\mathrm{AIH}^{\mathrm{b}}-\mathrm{APC}$} & \multirow[t]{2}{*}{$\mathrm{AIH}^{\mathrm{b}}$-KRAS } & \multirow[t]{2}{*}{$\mathrm{AIH}^{\mathrm{b}}-\mathrm{TP} 53$} \\
\hline & & & & $A P C$ mut $^{\mathrm{a}}$ & $K R A S$ mut $^{\mathrm{a}}$ & $T P 53 \mathrm{mut}^{\mathrm{a}}$ & & & & & \\
\hline \multirow[t]{2}{*}{18} & III & 46 & A & + & - & - & 1 & 2,2 & + & + & ND \\
\hline & & & $\mathrm{B}$ & + & + & - & 45 & 97,8 & & & \\
\hline \multirow[t]{2}{*}{21} & II & 50 & A & - & + & - & 2 & 4 & + & - & ND \\
\hline & & & B & + & + & - & 48 & 96 & & & \\
\hline 57 & III & 50 & $\mathrm{~A}$ & + & + & - & 50 & 100 & - & - & ND \\
\hline \multirow[t]{4}{*}{$8^{\mathrm{c}}$} & III & 50 & $\mathrm{~A}$ & $+/+$ & - & - & 3 & 6 & + & ND & + \\
\hline & & & $\mathrm{B}$ & - & - & + & 9 & 18 & & & \\
\hline & & & $\mathrm{C}$ & + & - & + & 8 & 16 & & & \\
\hline & & & $\mathrm{D}$ & $+/+$ & - & + & 30 & 60 & & & \\
\hline 9 & IV & 50 & A & + & - & + & 50 & 100 & + & ND & - \\
\hline \multirow[t]{3}{*}{17} & III & 47 & A & + & - & - & 1 & 2,1 & + & ND & + \\
\hline & & & $\mathrm{B}$ & - & - & + & 3 & 6,4 & & & \\
\hline & & & $\mathrm{C}$ & + & - & + & 43 & 91,5 & & & \\
\hline \multirow[t]{3}{*}{20} & II & 42 & A & + & - & - & 4 & 9,5 & + & ND & + \\
\hline & & & B & - & - & + & 3 & 7,1 & & & \\
\hline & & & $\mathrm{C}$ & + & - & + & 35 & 83,3 & & & \\
\hline 28 & II & 50 & A & + & - & + & 50 & 100 & - & ND & + \\
\hline 36 & I & 50 & A & + & - & + & 50 & 100 & + & ND & + \\
\hline \multirow[t]{2}{*}{39} & III & 50 & A & - & - & + & 38 & 76 & - & ND & + \\
\hline & & & B & + & - & + & 12 & 24 & & & \\
\hline \multirow[t]{3}{*}{$43^{c}$} & I & 50 & A & + & - & - & 2 & 4 & + & ND & + \\
\hline & & & B & + & - & + & 2 & 4 & & & \\
\hline & & & $\mathrm{C}$ & $+/+$ & - & + & 46 & 92 & & & \\
\hline \multirow[t]{2}{*}{$46^{c}$} & II & 50 & A & + & - & - & 5 & 10 & + & ND & + \\
\hline & & & B & + & - & $+/+$ & 45 & 90 & & & \\
\hline \multirow[t]{2}{*}{48} & III & 41 & A & - & - & + & 2 & 4,9 & + & ND & - \\
\hline & & & B & + & - & + & 39 & 95,1 & & & \\
\hline \multirow[t]{2}{*}{$63^{c}$} & II & 50 & A & $+/+$ & - & - & 1 & 2 & + & ND & + \\
\hline & & & B & $+/+$ & - & + & 49 & 98 & & & \\
\hline 66 & I & 50 & A & + & - & + & 50 & 100 & + & ND & + \\
\hline 67 & III & 49 & A & - & - & + & 18 & 36,7 & + & ND & + \\
\hline & & & B & + & - & + & 31 & 63,3 & & & \\
\hline 70 & III & 44 & A & + & - & - & 2 & 4,5 & + & ND & + \\
\hline & & & B & + & - & + & 42 & 95,5 & & & \\
\hline 75 & III & 40 & A & + & - & - & 5 & 12,5 & + & ND & + \\
\hline & & & B & + & - & + & 35 & 87,5 & & & \\
\hline 76 & II & 40 & A & + & - & - & 2 & 5 & + & ND & + \\
\hline & & & B & - & - & + & 7 & 17,5 & & & \\
\hline & & & $\mathrm{C}$ & + & - & + & 31 & 77,5 & & & \\
\hline 10 & II & 49 & A & - & - & + & 5 & 10,2 & ND & + & - \\
\hline & & & B & - & + & + & 44 & 89,8 & & & \\
\hline 26 & II & 42 & A & - & + & - & 4 & 9,5 & ND & + & + \\
\hline & & & B & - & - & + & 9 & 21,4 & & & \\
\hline & & & $\mathrm{C}$ & - & + & + & 29 & 69 & & & \\
\hline 47 & III & 50 & A & - & + & + & 50 & 100 & ND & - & + \\
\hline
\end{tabular}


Table 1 continued

\begin{tabular}{|c|c|c|c|c|c|c|c|c|c|c|c|}
\hline \multirow[t]{2}{*}{ Case } & \multirow[t]{2}{*}{ Clinical stage } & \multirow[t]{2}{*}{ Samples/tumor } & \multirow[t]{2}{*}{ Clone } & \multicolumn{3}{|l|}{ Genotype } & \multirow[t]{2}{*}{ Areas } & \multirow[t]{2}{*}{$\%$} & \multirow[t]{2}{*}{$\mathrm{AIH}^{\mathrm{b}}-\mathrm{APC}$} & \multirow[t]{2}{*}{ AIH ${ }^{\mathrm{b}}-\mathrm{KRAS}$} & \multirow[t]{2}{*}{$\mathrm{AIH}^{\mathrm{b}}-\mathrm{TP} 53$} \\
\hline & & & & $A P C \mathrm{mut}^{\mathrm{a}}$ & $K R A S$ mut $^{\mathrm{a}}$ & TP53 $\mathrm{mut}^{\mathrm{a}}$ & & & & & \\
\hline \multirow[t]{2}{*}{64} & I & 46 & A & - & + & - & 23 & 50 & ND & + & + \\
\hline & & & B & - & + & + & 23 & 50 & & & \\
\hline
\end{tabular}

${ }^{\mathrm{a}}$ mut mutations given in Table $\mathrm{S} 1$ for each tumor case

b $A I H$ heterogeneity in allelic imbalance

c Cases with two mutations in one gene (case 8, 43, 46, 49, 63). "+/+" means two mutations, "+" one mutation, "-" no mutation in the gene. For case 8 single mutation in APC is c. $3139 \mathrm{G}>\mathrm{T}$; for case 43-c.904 C>T

populations. To detect mutations, we used the SNaPshot assay, which is a quantitative primer extension assay that yields ratios of mutated and normal alleles.

All 42 samples were subjected to multipoint microsampling. Figure 1 shows a microscopic image of a tumor tissue section from case 12 (Fig. 1a), an electropherogram of the SNaPshot assay (Fig. 1b) and a graphical presentation of the mutant allele ratios $(\triangle \mathrm{M})$ of $A P C$ and $K R A S$ (Fig. 1c). Although all five regions in Fig. 1a contained cells with mutations in $A P C$, not all of the regions carried mutations in KRAS; there was only one peak (ddG) for the $K R A S$ allele in region 2 (Fig. 1b, c).

The mutation statuses of all samples taken from primary tumor tissues are summarized in Table 1. Representative results of areas from primary colorectal cancer tissues plotted in three dimensions were created using the $\triangle \mathrm{M}$ values of $A P C$, $K R A S$ and TP53, and 2-D plots were made for the samples with two mutated genes. These results are shown in Fig. S1.

There was usually more than one subpopulation in each primary tumor. Indeed, only two of $15(13.3 \%)$ cases with three gene mutations and eight of $27(29.6 \%)$ cases with two gene mutations exhibited a single subpopulation. In most heterogeneous tumors, cells with all mutations constituted the major population (Table 1).

Because the SNaPshot assay measures relative levels of both alleles, we also revealed heterogeneity of allelic imbalance. Because the standard deviation of the SNaPshot assay was 0.08 [14], cases where the data ranges were over 0.32 $( \pm 2 \sigma)$ were considered heterogeneous. For example, case 10 showed heterogeneity in the allele ratio of $K R A S(\Delta \mathrm{M}=$ $0.0-0.53)$, but not of TP53 $(\Delta \mathrm{M}=0.94-1.0)$, whereas case 6 did not show heterogeneity for either of the two genes $(\Delta \mathrm{M}=0.46-0.61$ for KRAS and 0.85-1.0 for APC) (Fig. S1). The status of the heterogeneity of allelic imbalance is shown in Table 1 . The heterogeneity was probably due to a mixture of two or more cell subpopulations with different allelic ratios.

Comparison of intratumor genetic heterogeneity between primary and metastatic tumors

Among the 15 primary tumors with the three gene mutations, six cases had corresponding hepatic metastatic tumors. The details of mutations revealed by bulk tissue analysis are shown in Table 2. Except for two cases (82 and 85), metastatic tissues carried the same mutations as the corresponding primary tumors. We compared the intratumor heterogeneity of the three gene mutations between primary and metastatic tumors for each case. Table 3 summarizes the mutation status of subpopulations in the tissue pairs. The percentages of each subpopulation in a primary tumor and its paired metastasis are graphically presented for all six cases in Fig. 2.
Table 2 Mutations found by bulk tissue analysis of six pairs of primary and metastatic tumors

a Stop codon

\begin{tabular}{|c|c|c|c|c|}
\hline Case & Sample & $A P C$ mutation & KRAS mutation & TP53 mutation \\
\hline \multirow[t]{2}{*}{1} & $\mathrm{~T}$ & c. $4348 \mathrm{C}>\mathrm{T}$ R $1450^{\mathrm{a}}$ & c. $34 \mathrm{G}>\mathrm{T} \mathrm{G} 12 \mathrm{C}$ & c. $818 \mathrm{G}>\mathrm{A}$ R $273 \mathrm{H}$ \\
\hline & M & c. $4348 \mathrm{C}>\mathrm{T}$ R $1450^{\mathrm{a}}$ & c. $34 \mathrm{G}>\mathrm{T}$ G12C & c. $818 \mathrm{G}>\mathrm{A}$ R $273 \mathrm{H}$ \\
\hline \multirow[t]{2}{*}{51} & $\mathrm{~T}$ & c. $2626 \mathrm{C}>\mathrm{T} \mathrm{R} 876^{\mathrm{a}}$ & c. $35 \mathrm{G}>\mathrm{T} \mathrm{G} 12 \mathrm{~V}$ & c. $818 \mathrm{G}>\mathrm{A} \mathrm{R} 273 \mathrm{H}$ \\
\hline & M & c. $2626 \mathrm{C}>\mathrm{T} \mathrm{R} 876^{\mathrm{a}}$ & c. $35 \mathrm{G}>\mathrm{T} \mathrm{G} 12 \mathrm{~V}$ & c. $818 \mathrm{G}>\mathrm{A}$ R $273 \mathrm{H}$ \\
\hline \multirow[t]{2}{*}{81} & $\mathrm{~T}$ & c. $1690 \mathrm{C}>\mathrm{T} \mathrm{R}^{2} 54^{\mathrm{a}}$ & c.183 A>T Q61H & c. $524 \mathrm{G}>\mathrm{A}$ R $175 \mathrm{H}$ \\
\hline & M & c. $1690 \mathrm{C}>\mathrm{T} \mathrm{R} 564^{\mathrm{a}}$ & c.183 A>T Q61H & c. $524 \mathrm{G}>\mathrm{A}$ R $175 \mathrm{H}$ \\
\hline \multirow[t]{2}{*}{82} & $\mathrm{~T}$ & c. $2626 \mathrm{C}>\mathrm{T} \mathrm{R} 876^{\mathrm{a}}$ & c. $34 \mathrm{G}>\mathrm{A} \mathrm{G} 12 \mathrm{~S}$ & c.755_63 del 9 bp \\
\hline & M & - & c. $34 \mathrm{G}>\mathrm{A} \mathrm{G} 12 \mathrm{~S}$ & c.755_63 del 9 bp \\
\hline \multirow[t]{2}{*}{83} & $\mathrm{~T}$ & c.4147 insA & c.38 G>A G13D & c. 682 ins 8 bp \\
\hline & M & c.4147 insA & c. $38 \mathrm{G}>\mathrm{A}$ G13D & c. 682 ins 8 bp \\
\hline \multirow[t]{2}{*}{85} & $\mathrm{~T}$ & c. $2626 \mathrm{C}>\mathrm{T} \mathrm{R} 876^{\mathrm{a}}$ & c. $35 \mathrm{G}>\mathrm{T} \mathrm{G} 12 \mathrm{~V}$ & c. $733 \mathrm{G}>\mathrm{A}$ G245S \\
\hline & M & - & c. $35 \mathrm{G}>\mathrm{T} \mathrm{G} 12 \mathrm{~V}$ & c. $733 \mathrm{G}>\mathrm{A}$ G245S \\
\hline
\end{tabular}


Table 3 Cell populations found in primary tumors and metastases of the patients

\begin{tabular}{|c|c|c|c|c|c|c|c|c|c|c|}
\hline \multirow[t]{2}{*}{ Case } & \multirow[t]{2}{*}{ Clone } & \multicolumn{3}{|l|}{ Genotype } & \multirow{2}{*}{$\begin{array}{l}\text { Examined } \\
\text { samples/ } \\
\text { tumor }\end{array}$} & \multirow{2}{*}{$\begin{array}{l}\text { Areas in } \\
\text { primary } \\
\text { tumor }^{b}\end{array}$} & \multirow[t]{2}{*}{$\%$} & \multirow{2}{*}{$\begin{array}{l}\text { Examined } \\
\text { samples/ } \\
\text { metastasis }\end{array}$} & \multirow{2}{*}{$\begin{array}{l}\text { Areas in } \\
\text { metastasis }\end{array}$} & \multirow[t]{2}{*}{$\%$} \\
\hline & & $\mathrm{APC}_{\text {mut }}{ }^{\mathrm{a}}$ & KRAS mut ${ }^{\mathrm{a}}$ & TP53 mut $^{\mathrm{a}}$ & & & & & & \\
\hline \multirow[t]{3}{*}{1} & A & - & - & c. $818 \mathrm{G}>\mathrm{A}$ R $273 \mathrm{H}$ & 42 & 3 & 7.1 & 50 & 0 & 0 \\
\hline & $\mathrm{B}$ & c. 4348 C $>$ T R $1450^{\text {c }}$ & - & c. $818 \mathrm{G}>\mathrm{A}$ R273H & & 1 & 2.4 & & 36 & 72.0 \\
\hline & $\mathrm{C}$ & c. $4348 \mathrm{C}>\mathrm{T} \mathrm{R} 1450^{\mathrm{c}}$ & c. $34 \mathrm{G}>\mathrm{T} \mathrm{G} 12 \mathrm{C}$ & c. $818 \mathrm{G}>\mathrm{A}$ R273H & & 38 & 90.5 & & 14 & 28.0 \\
\hline \multirow[t]{3}{*}{51} & A & - & - & c. $818 \mathrm{G}>\mathrm{A}$ R273H & 42 & 1 & 2.4 & 50 & 0 & 0 \\
\hline & B & c. $2626 \mathrm{C}>\mathrm{T} \mathrm{R} 876^{\mathrm{c}}$ & - & c. $818 \mathrm{G}>\mathrm{A}$ R273H & & 2 & 4.8 & & 0 & 0 \\
\hline & $\mathrm{C}$ & c. $2626 \mathrm{C}>\mathrm{T} \mathrm{R} 876^{\mathrm{c}}$ & c. $35 \mathrm{G}>\mathrm{T} \mathrm{G} 12 \mathrm{~V}$ & c. $818 \mathrm{G}>\mathrm{A}$ R273H & & 39 & 92.9 & & 50 & 100.0 \\
\hline \multirow[t]{2}{*}{81} & A & c. $1690 \mathrm{C}>\mathrm{T} \mathrm{R} 564^{\mathrm{c}}$ & c.183 A>T Q61H & - & 42 & 4 & 9.5 & 50 & 7 & 14.0 \\
\hline & $\mathrm{B}$ & c. $1690 \mathrm{C}>\mathrm{T} \mathrm{R} 564^{\mathrm{c}}$ & c.183 A>T Q61H & c. $524 \mathrm{G}>\mathrm{A}$ R175H & & 38 & 90.5 & & 43 & 86.0 \\
\hline \multirow[t]{4}{*}{82} & A & c. $2626 \mathrm{C}>\mathrm{T} \mathrm{R} 876^{\mathrm{c}}$ & - & c. $755 \_63$ del 9 bp & 48 & 1 & 2.1 & 49 & 0 & 0 \\
\hline & $\mathrm{B}$ & - & - & c.755_63 del 9 bp & & 3 & 6.3 & & 25 & 51.0 \\
\hline & $\mathrm{C}$ & - & c. $34 \mathrm{G}>\mathrm{A} \mathrm{G} 12 \mathrm{~S}$ & c.755_63 del 9 bp & & 41 & 85.4 & & 24 & 49.0 \\
\hline & $\mathrm{D}$ & c. $2626 \mathrm{C}>\mathrm{T} \mathrm{R} 876^{\mathrm{c}}$ & c. $34 \mathrm{G}>\mathrm{A} \mathrm{G} 12 \mathrm{~S}$ & c. $755 \_63$ del 9 bp & & 3 & 6.3 & & 0 & 0 \\
\hline \multirow[t]{2}{*}{83} & A & c.4147 insA & c. $38 \mathrm{G}>\mathrm{A}$ G13D & - & 45 & 4 & 8.9 & 50 & 0 & 0 \\
\hline & B & c. 4147 insA & c.38 G>A G13D & c. 682 ins 8 bp & & 41 & 91.1 & & 50 & 100.0 \\
\hline \multirow[t]{5}{*}{85} & A & c. $2626 \mathrm{C}>\mathrm{T} \mathrm{R} 876^{\mathrm{c}}$ & - & c.733 G>A G245S & 44 & 1 & 2.3 & 50 & 0 & 0 \\
\hline & B & - & c. $35 \mathrm{G}>\mathrm{T} \mathrm{G} 12 \mathrm{~V}$ & - & & 2 & 4.5 & & 0 & 0 \\
\hline & $\mathrm{C}$ & - & - & c.733 G>A G245S & & 1 & 2.3 & & 9 & 18.0 \\
\hline & $\mathrm{D}$ & - & c. $35 \mathrm{G}>\mathrm{T} \mathrm{G} 12 \mathrm{~V}$ & c.733 G>A G245S & & 35 & 79.5 & & 41 & 82.0 \\
\hline & $\mathrm{E}$ & c. $2626 \mathrm{C}>\mathrm{T} \mathrm{R} 876^{\mathrm{c}}$ & c. $35 \mathrm{G}>\mathrm{T} \mathrm{G} 12 \mathrm{~V}$ & c.733 G>A G245S & & 5 & 11.4 & & 0 & 0 \\
\hline
\end{tabular}

a mut mutation

${ }^{b}$ Number of samples with a specific genotype

c Stop codon

In cases 82 and 85 , there were discrepancies of mutation patterns; both cases lacked APC mutations in metastatic tumors (Table 2). Indeed, multipoint microsampling revealed that metastatic tumors lacked subpopulations with an $A P C$ mutation in both cases (i.e., subpopulations $\mathrm{A}$ and $\mathrm{D}$ in case 82 and subpopulations $\mathrm{A}$ and $\mathrm{E}$ in case 85 ) (Table 3 and Fig. 2d and f).

In cases 51 and 83, the major subpopulation of the primary tumor occupied all areas of the metastatic tumor (Fig. $2 b$ and $\mathrm{e}$, respectively). This result suggests that at least most cancer cells in the liver metastases were derived from the major subpopulations of the primary tumors in these two cases. In other cases, there was more than one subpopulation within the metastatic tumor tissue. In all cases, there were no de novo mutations, which suggests that cells from subpopulations in the primary tumors moved to the liver. In cases 1,82 and 85 , the proportions of KRAS mutation-negative subpopulations increased (Fig. 2a, d and f, respectively). Because both the primary and metastatic tumors in case 1 were mostly composed of a single major subpopulation, we performed arrayCGH to exclude the possibility that the KRAS mutant allele would be lost by LOH after metastasizing. Notably, there was no LOH in the KRAS region (data not shown), which suggests that the mutation-negative subpopulation of the metastatic tumor was derived from the minor mutation-negative subpopulation of the primary tumor.

\section{Discussion}

Multipoint microsampling offers much higher resolution than techniques used in similar studies in the literature [2-11]. Consequently, we found intratumor heterogeneity in $87 \%$ of primary tumors with mutations in three genes (i.e., APC, KRAS and TP53) and in $70 \%$ of tumors with mutations in two of the three genes. We then compared mutation statuses between primary and metastatic tumors. The multipoint microsampling approach revealed that discrepancies of mutation patterns found by bulk tissue analysis were due to loss of subpopulations in the metastatic tissues. In addition, multipoint microsampling uncovered substantial changes in subpopulations that were not detected by bulk tissue analysis, such as the increase of KRAS mutation-negative cells in metastatic tumors.

A recent study of exome sequencing in pancreatic cancer reported a similar finding. Indeed, clonal populations that give rise to distant metastases were represented in one of the subpopulations taken from several parts of the 
Fig. 2 Genetic heterogeneity in six pairs of primary and metastatic tumor tissues. The percentages of areas with a particular mutation status in the primary tumor (PT) and its paired metastasis (M) are graphically presented for each case. The mutation patterns are: $\mathrm{APC}^{\mathrm{N}} \mathrm{KRAS}^{\mathrm{N}} \mathrm{TP}^{\mathrm{M}} 3^{\mathrm{M}}$, yellow; $\mathrm{APC}^{\mathrm{N}} \mathrm{KRAS}^{\mathrm{M}} \mathrm{TP}^{\mathrm{N}}{ }^{\mathrm{N}}$, light purple; $\mathrm{APC}^{\mathrm{M}} \mathrm{KRAS}^{\mathrm{N}} \mathrm{TP}^{\mathrm{M}}{ }^{\mathrm{M}}$, dark blue;

$\mathrm{APC}^{\mathrm{N}} \mathrm{KRAS}^{\mathrm{M}} \mathrm{TP}^{\mathrm{T}}{ }^{\mathrm{M}}$, green; $\mathrm{APC}^{\mathrm{M}} \mathrm{KRAS}^{\mathrm{M}} \mathrm{TP}^{\mathrm{N}}{ }^{\mathrm{N}}$, light blue; and $\mathrm{APC}^{\mathrm{M}} \mathrm{KRAS}^{\mathrm{M}} \mathrm{TP}^{\mathrm{M}}{ }^{\mathrm{M}}$, red $(\mathrm{M}$, mutated gene; $\mathrm{N}$, no mutation). a Case 1 , b case 51 , c case $81, \mathbf{d}$ case $82, \mathbf{e}$ case 83 , and $\mathbf{f}$ case 85
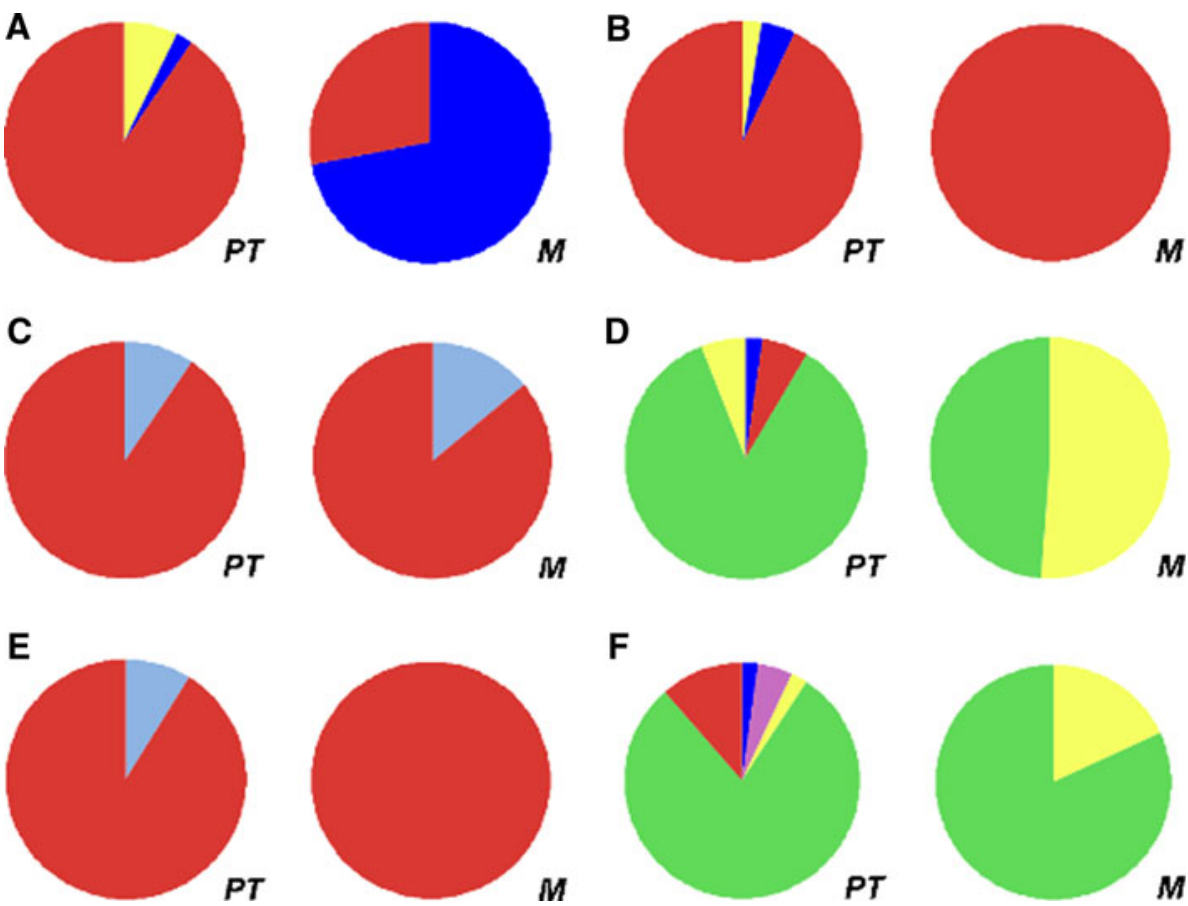

$\mathbf{F}$

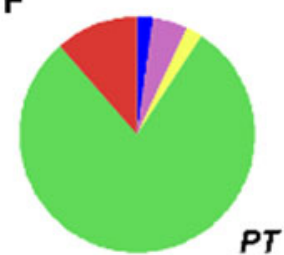

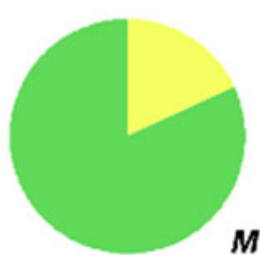

primary tumors [15]. This study by Campbell et al. identified far more mutations than our study. In addition, the Campbell et al. study identified the original parental nonmetastatic clones from which metastatic clones evolved. In the present study, we used multipoint microsampling, which has a markedly greater spatial resolution because the exome sequencing study was based on DNA prepared from more than 100 million cells. We found that the metastatic tumors included more than one subpopulation of the primary tumor in four cases (Fig. 2). In liver metastases, colorectal cancer cells move to the liver via the portal vein, which differs from ordinary distant metastases where cells move through the systemic circulation. The simultaneous migration of more than one subpopulation might be specific to liver metastases of colorectal cancer because of this anatomical characteristic.

Clinical trials with cetuximab or panitumumab revealed that patients with wild-type $K R A S$ responded to therapy (up to $50 \%$ ), whereas those with tumors exhibiting KRAS mutations had a low response rate (0-6\%) [16-21]. Although the KRAS mutation status is predictive of cetuximab/panitumumab efficacy, there are cases in which the response did not match the prediction. Based on a hypothesis that the heterogeneity of KRAS mutations between primary and metastatic tumors may be responsible for this discrepancy, Italiano et al. analyzed 95 pairs of primary and metastatic tumors. Italiano et al. found that $K R A S$ was mutated in primary tumors in six cases, but wild-type $K R A S$ was found in metastatic tumors. In addition, eight cases showed that KRAS was mutated in metastatic tumors, but wild-type KRAS was found in primary tumors [22]. Moreover, Baldus et al. analyzed 20 pairs of a primary tumor and a distant metastasis, and found heterogeneity of the KRAS mutation status in two cases [23]. Heterogeneity between primary and lymph node metastases was more frequent ( $31 \%$ of cases) than that between primary and metastatic tumors. Both studies indicated that the discrepancy of the KRAS mutation status between a primary tumor and its distant metastasis was likely to be too rare to account for the discordance between the KRAS mutation status and cetuximab/panitumumab efficacy. The results from these two studies, however, were obtained with analysis of bulk tissues or macrodissection. The present study demonstrated that there were changes in proportions of subpopulations that could not be detected by bulk tissue analysis. Thus, the possibility remains that heterogeneity of the KRAS mutation status could be the cause of altered cetuximab/panitumumab efficacy. Our preliminary study on intratumor heterogeneity of EGFR mutations in lung cancer suggested that tumors that have cancer cells with wild-type EGFR exhibited an inferior response to gefitinib [11]. Because molecularly targeted drugs are becoming the mainstream of adjuvant therapy, intratumor heterogeneity of target genes might be an important factor requiring further intensive analysis.

Acknowledgments The authors thank Dr Kazuya Taniguchi for technical advice. This work was partly supported by the Knowledge Cluster Initiative (the Keihanna Science City area) of the Ministry of Education, Culture, Sports, Science, and Technology of Japan.

Open Access This article is distributed under the terms of the Creative Commons Attribution Noncommercial License which 
permits any noncommercial use, distribution, and reproduction in any medium, provided the original author(s) and source are credited.

\section{References}

1. Visvader JE, Lindeman GJ (2008) Cancer stem cells in solid tumours: accumulating evidence and unresolved questions. Nat Rev Cancer 8(10):755-768

2. Baisse B, Bouzourene H, Saraga EP et al (2001) Intratumor genetic heterogeneity in advanced human colorectal adenocarcinoma. Int J Cancer 93(3):346-352

3. Barnetson R, Jass J, Tse R et al (2000) Mutations associated with microsatellite unstable colorectal carcinomas exhibit widespread intratumoral heterogeneity. Genes Chromosomes Cancer 29(2): 130-136

4. Giaretti W, Rapallo A, Sciutto A et al (2000) Intratumor heterogeneity of k-ras and p53 mutations among human colorectal adenomas containing early cancer. Anal Cell Pathol 21(2):49-57

5. Gonzalez-Garcia I, Sole RV, Costa J (2002) Metapopulation dynamics and spatial heterogeneity in cancer. Proc Natl Acad Sci USA 99(20):13085-13089

6. Konishi N, Hiasa Y, Matsuda $\mathrm{H}$ et al (1995) Intratumor cellular heterogeneity and alterations in ras oncogene and p53 tumor suppressor gene in human prostate carcinoma. Am J Pathol 147 (4):1112-1122

7. Losi L, Baisse B, Bouzourene $\mathrm{H}$ et al (2005) Evolution of intratumoral genetic heterogeneity during colorectal cancer progression. Carcinogenesis 26(5):916-922

8. Lyng H, Beigi M, Svendsrud DH et al (2004) Intratumor chromosomal heterogeneity in advanced carcinomas of the uterine cervix. Int J Cancer 111(3):358-366

9. Samowitz WS, Slattery ML (1999) Regional reproducibility of microsatellite instability in sporadic colorectal cancer. Genes Chromosomes Cancer 26(2):106-114

10. Takeshima Y, Amatya VJ, Daimaru Y et al (2001) Heterogeneous genetic alterations in ovarian mucinous tumors: application and usefulness of laser capture microdissection. Hum Pathol 32(11): 1203-1208

11. Taniguchi K, Okami J, Kodama K et al (2008) Intratumor heterogeneity of epidermal growth factor receptor mutations in lung cancer and its correlation to the response to gefitinib. Cancer Sci 99(5):929-935

12. Marusyk A, Polyak K (2010) Tumor heterogeneity: causes and consequences. Biochim Biophys Acta 1805(1):105-117

13. Fearon ER, Vogelstein B (1990) A genetic model for colorectal tumorigenesis. Cell 61(5):759-767

14. Goranova TE, Ohue M, Kato K (2009) Putative precursor cancer cells in human colorectal cancer tissue. Int J Clin Exp Pathol 2(2):154-162

15. Campbell PJ, Yachida S, Mudie LJ et al (2010) The patterns and dynamics of genomic instability in metastatic pancreatic cancer. Nature 467(7319):1109-1113

16. De Roock W, Piessevaux H, De Schutter J et al (2008) KRAS wild-type state predicts survival and is associated to early radiological response in metastatic colorectal cancer treated with cetuximab. Ann Oncol 19(3):508-515

17. Di Fiore F, Blanchard F, Charbonnier F et al (2007) Clinical relevance of KRAS mutation detection in metastatic colorectal cancer treated by Cetuximab plus chemotherapy. Br J Cancer 96(8):1166-1169

18. Hecht JR, Patnaik A, Berlin J et al (2007) Panitumumab monotherapy in patients with previously treated metastatic colorectal cancer. Cancer 110(5):980-988

19. Khambata-Ford S, Garrett CR, Meropol NJ et al (2007) Expression of epiregulin and amphiregulin and K-ras mutation status predict disease control in metastatic colorectal cancer patients treated with cetuximab. J Clin Oncol 25(22):3230-3237

20. Lievre A, Bachet JB, Boige V et al (2008) KRAS mutations as an independent prognostic factor in patients with advanced colorectal cancer treated with cetuximab. J Clin Oncol 26(3): 374-379

21. Lievre A, Bachet JB, Le Corre D et al (2006) KRAS mutation status is predictive of response to cetuximab therapy in colorectal cancer. Cancer Res 66(8):3992-3995

22. Italiano A, Hostein I, Soubeyran I et al (2010) KRAS and BRAF mutational status in primary colorectal tumors and related metastatic sites: biological and clinical implications. Ann Surg Oncol 17(5):1429-1434

23. Baldus SE, Schaefer KL, Engers R et al (2010) Prevalence and heterogeneity of KRAS, BRAF, and PIK3CA mutations in primary colorectal adenocarcinomas and their corresponding metastases. Clin Cancer Res 16(3):790-799 